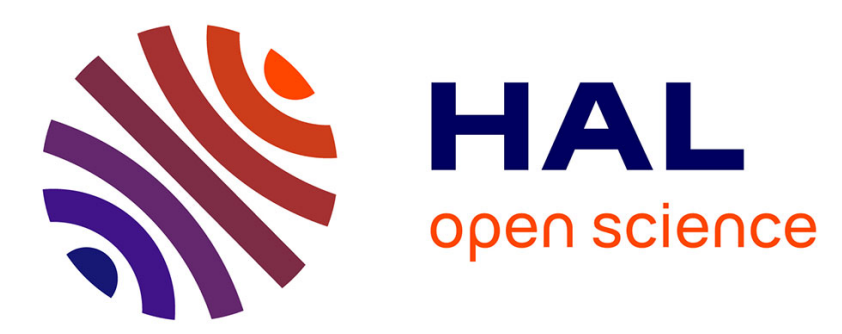

\title{
Predicting viscoplastic anisotropy in the upper mantle: a comparison between experiments and polycrystal plasticity models
}

Lucan Mameri, Andrea Tommasi, Javier Signorelli, Lars Hestbjerg Hansen

\section{- To cite this version:}

Lucan Mameri, Andrea Tommasi, Javier Signorelli, Lars Hestbjerg Hansen. Predicting viscoplastic anisotropy in the upper mantle: a comparison between experiments and polycrystal plasticity models. Physics of the Earth and Planetary Interiors, 2019, 286, pp.69- 80. 10.1016/j.pepi.2018.11.002 . hal01933565

\section{HAL Id: hal-01933565 https://hal.science/hal-01933565}

Submitted on 23 Nov 2018

HAL is a multi-disciplinary open access archive for the deposit and dissemination of scientific research documents, whether they are published or not. The documents may come from teaching and research institutions in France or abroad, or from public or private research centers.
L'archive ouverte pluridisciplinaire HAL, est destinée au dépôt et à la diffusion de documents scientifiques de niveau recherche, publiés ou non, émanant des établissements d'enseignement et de recherche français ou étrangers, des laboratoires publics ou privés. 


\title{
Predicting viscoplastic anisotropy in the upper mantle: a comparison between experiments and polycrystal plasticity models
}

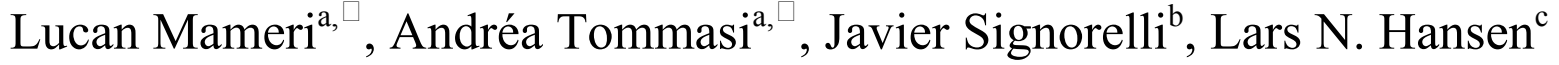 \\ ${ }^{a}$ Géosciences Montpellier - CNRS \& Université de Montpellier, France \\ b Instituto de Física de Rosario, CONICET \& Universidad Nacional de Rosario, Argentina \\ ${ }^{\mathrm{c}}$ Department of Earth Sciences, University of Oxford, United Kingdom \\ Corresponding authors: \\ Lucan Mameri \\ lucan.mameri@gm.univ-montp2.fr \\ Andréa Tommasi \\ andrea.tommasi@umontpellier.fr
}

The published version of this paper can be found as:

Mameri, L., Tommasi, A., Signorelli, J., Hansen, L. N. (2019). Predicting viscoplastic anisotropy in the upper mantle: a comparison between experiments and polycrystal plasticity models. Phys. Earth Planet.

Inter. https://doi.org/10.1016/j.pepi.2018.11.002

The supplementary material can be found online or under request. 


\begin{abstract}
Viscoplastic deformation of mantle rocks produces crystal preferred orientations ( $\mathrm{CPO}$ or texture) of olivine and, by consequence, an anisotropic mechanical response for subsequent deformation events. Olivine polycrystals deformed experimentally in torsion and then in extension normal to the previous shear plane have ex- tensional strengths $<2$ times higher than the torsional ones. Implementation of this viscoplastic anisotropy in geodynamic codes depends nevertheless on the predictions of polycrystal plasticity models, since the full range of orientation relations between applied stresses and the broad range of texture types observed in upper mantle rocks may only be tested numerically. Here, we compare instantaneous viscoplastic responses of olivine polycrystals predicted by tangent and secondorder viscoplastic self-consistent (VPSC) and stress equilibrium-based models to experimental data. These polycrystal plasticity models, in which only dislocation glide is considered to accommodate strain, reproduce qualitatively the viscoplastic anisotropy observed in the laboratory, but overestimate its magnitude for rocks with strong textures. The lower bound model better approaches the laboratory results, suggesting that the discrepancy between laboratory and numerical experiments arises from the models forcing strain compatibility in olivine polycrystals that can only deform by dislocation creep. The experimental data is indeed well reproduced by the second-order VPSC model modified to include additional isotropic deformation mechanisms, which accommodate the deformation components that cannot be produced by dislocation glide but do not produce plastic spin. These results corroborate that other processes in addition to dislocation glide contribute significantly to viscoplastic deformation under laboratory conditions, reducing the magnitude of anisotropy. In nature, coarser grain sizes probably result in lower activity of these additional deformation processes. We therefore propose that standard and modified second-order VPSC models provide a good prediction of the possible range of texture-induced viscoplastic anisotropy in upper mantle domains deforming by dislocation creep.
\end{abstract}

Keywords: olivine; crystal preferred orientation ; mechanical anisotropy; dislocation creep ; mantle deformation; rheology 


\section{Introduction}

The lithospheric mantle is predominantly composed of olivine, a mineral that displays highly anisotropic elastic and viscoplastic mechanical behavior (Kumazawa and Anderson, 1969; Bai et al., 1991). Upper mantle deformation by viscoplastic processes produces crystallographic preferred orientation (CPO or texture) of olivine (Nicolas and Christensen, 1987). These CPO result in anisotropic behavior at the scale of the rock (cm- to m-scale) and larger, as indicated by observations of coherent seismic anisotropy patterns correlated with the tectonic fabric of continental plates at the scale of hundreds of $\mathrm{km}$ (cf. review in Tommasi and Vauchez, 2015). Large-scale olivine CPO in the upper mantle has therefore been proposed as a source of structural memory in the upper mantle, leading to strain localization along shear zones formed during previous tectonic events (Vauchez et al., 1998; Tommasi and Vauchez, 2001).

The consequences of upper mantle viscoplastic anisotropy on the deformation of both the plates and convective mantle have been analyzed in a variety of numerical models. Many of these models used a simplified description of this anisotropy by imposing a constant difference between 'shear' and 'normal' viscosities and reorienting the viscosity tensor in response to ow based on a director vector approach (e.g., Honda, 1986; Christensen, 1987; Lev and Hager, 2008, 2011). These models, which tested normal to shear viscosity ratios of $10-100$, concluded that viscous anisotropy does change mantle flow patterns by stabilizing the boundary layers and enhancing strain localization. Most recent models have coupled polycrystal plasticity simulations into finite-element models; this allows the follow-up of the evolution of the orientation and intensity of the full viscosity tensor (e.g., Chastel et al., 1993; Knoll et al., 2009; Tommasi et al., 2009; Castelnau et al., 2009; Blackman et al., 2017). These more sophisticated models support the early conclusions on the role of viscous anisotropy on the stability of convection (Chastel et al., 1993), and corroborate that the olivine CPO formed during preexisting deformation episodes and subsequently frozen into the lithospheric mantle may explain the reactivation of ancient continental-scale structures to form new plate boundaries (Knoll et al., 2009; Tommasi et al., 2009) and change the mantle flow pattern beneath spreading ridges (Castelnau et al., 2009, Blackman et al., 2017). However, at the time most of those calculations were carried out, no experimental data existed for corroborating the predictions of the polycrystal plasticity models.

Recently, laboratory experiments demonstrated that olivine aggregates with textures formed in torsion are $\sim 2$ times weaker for further deformation in torsion than for extension perpendicular to the previous shear plane (Hansen et al., 2012a). These experiments provided the first quantitative data for validating the predictions of viscoplastic anisotropy in olivine-rich rocks by polycrystal plasticity models. This validation is important because the use of geodynamical models to study the effects of an anisotropic rheology on Earth dynamics depends directly on our ability to predict the anisotropy of viscoplastic behavior associated with any combination of $\mathrm{CPO}$ and loading geometry. Only polycrystal plasticity models can test in a physically consistent way the full range of orientation relations between applied stresses and the broad range of texture types observed in upper-mantle rocks.

In this work, we test how well different polycrystal plasticity models simulate the viscoplastic anisotropy of olivine polycrystals measured in the laboratory by calculating the instantaneous mechanical response in simple shear and axial extension for polycrystals with the same CPO as the experimental samples and comparing these predictions to (1) the final stresses measured in the torsion experiments that produced the CPO and (2) to the peak stresses measured in axial extension experiments subsequent to torsion. The comparison between the polycrystal plasticity models and the experimental data allows us to quantify the 
contribution of other processes to the deformation of olivine polycrystals under laboratory conditions and propose modeling approaches that predict the possible range of texture-induced viscoplastic anisotropy in upper mantle domains deforming by dislocation creep.

\section{Methods}

\subsection{Modeling viscoplastic behavior and texture evolution}

We tested the capability of different polycrystal plasticity models to predict the viscoplastic behavior of textured olivine-rich rocks at high temperature and moderate pressure by simulating the torsion and ex- tension tests of Hansen et al. (2012a). Hansen et al. (2016a) presents complementary results (extension followed by torsion), but a heterogeneous distribution of strain in the initial extension experiments resulted in large variability among textures and stresses. For testing the different polycrystal plasticity models, we require higher precision data. Therefore in the present study, we focus solely on simulating experiments from Hansen et al. (2012a) in which torsion was followed by extension. To do so, we calculated the instantaneous mechanical response of four olivine polycrystals that have precisely the same $\mathrm{CPO}$ as those measured in the experimental samples. We also applied the same loading geometries as in the experiments: simple shear parallel to the previous shear deformation that created the texture or axial extension parallel to the previous torsional axis (Fig. 1). The employed models are viscoplastic and include no elasticity. This simplification is justified by fullfield simulations of polycrystalline materials performed in the elasto-viscoplastic regime that demonstrate that the viscoplastic behavior controls the mechanical response of the polycrystal even at very small strains $(<1 \%$, Lebensohn et al., 2012).

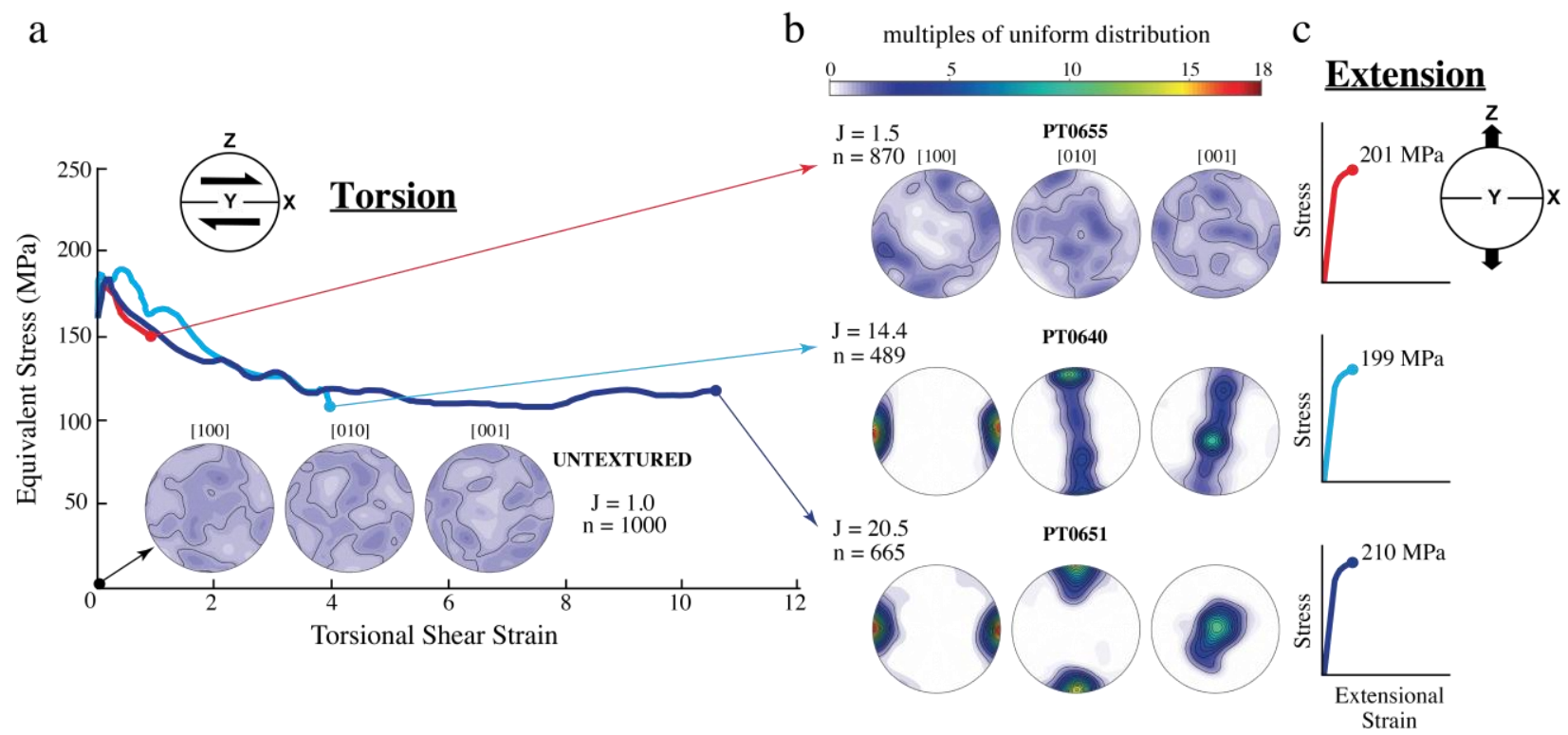

Fig. 1. (a) Stress-strain curves for torsion experiments by Hansen et al. (2012a). (b) CPO produced by the torsion experiments and used as initial textures in the extension experiments and in the polycrystal plasticity models. (c) Stress-strain curves for extension experiments by Hansen et al. (2012a). Instantaneous stresses predicted by the models in simple shear and extension are compared to (a) final stresses in torsion and (c) peak stresses in extension, respectively. The stresses in extension are averaged values normalized to a constant strain-rate of $2 \times 10^{-4} \mathrm{~s}^{-1}$. The number of grains in each CPO is indicated by the value $n$, and the CPO intensity by the value $J$ (J-index). Pole figures are contoured as multiples of uniform distribution; the same contour range limits are applied to all pole figures for easy evaluation of variations in $\mathrm{CPO}$ strength. 
The viscoplastic mechanical response of the olivine polycrystals to the imposed extensional or simple shear deformation has been modeled using three polycrystal plasticity models: the uniform stress (lower bound) approach (Sachs, 1928; Chastel et al., 1993), the tangent VPSC model (Lebensohn and Tomé, 1993), and the more recent second-order formulation of the VPSC model (Ponte Castañeda, 2002; Lebensohn et al., 2011). The three approaches differ in the way the crystal interactions are modeled. The lower bound assumes constant stress in all grains and disregards strain compatibility. In the VPSC approach, each grain is represented as a viscoplastic inclusion embedded in and interacting with a homogeneous viscoplastic medium that represents the average polycrystal behavior. Stress equilibrium and strain compatibility at the aggregate scale are obtained by approximating the macroscopic strain rate and stress to the average of strain rate and stress at the grain scale, respectively. The second-order (SO)- and tangent (TGT)-VPSC models differ in how the non-linear behavior of the material is estimated. Both formulations are based on the same linearization scheme, which considers heterogeneous stress and strain rate within the polycrystal. However, only the second-order formulation accounts for intragranular strain rate fluctuations to predict the effective polycrystal behavior (Ponte Castañeda, 2002; Lebensohn et al., 2011).

In all three approaches, individual crystals deform only by dislocation glide on a finite number of slip systems, which are a function of the crystal structure. The relative strength of these slip systems, that is, the critical resolved shear stress (CRSS) needed to activate dislocation glide on each system, depends on the temperature, pressure, and stress. For technical reasons, the experiments of Hansen et al. (2012a) were performed using olivine crystals enriched in iron $\left(\mathrm{Fo}_{50}\right)$ relative to typical mantle olivines $\left(\mathrm{Fo}_{90}\right)$, for which mechanical data are not available for creep of single crystals. In the present study, we use CRSSs (Table 1) derived from deformation of natural olivine single crystals $\left(\mathrm{Fo}_{90}\right)$ at high temperatures and low pressure (Bai et al., 1991). The choice of these CRSSs, although arbitrary, is justified by the analysis of the CPO produced in the torsion experiments of Hansen et al. (2012a), which is consistent with the dominant activation of the (010)[100] slip system. We also examined the effect of setting equal the values of CRSS for the (001)[100] and (010)[100] slip systems, as used in previous VPSC models studying olivine CPO evolution at high temperature and low pressure (e.g., Tommasi et al., 2000), and we observed negligible variations in terms of mechanical behavior compared to the present model setup. Laboratory experiments and atomistic modeling suggest nevertheless a change in the relative resistance between the different slip systems, favoring glide of dislocations with [001] Burgers vectors relative to those with [100] Burgers vectors at high pressure (e.g., Durinck et al., 2007; Raterron et al., 2007). Comparison of the seismic anisotropy predicted by polycrystal plasticity models with high and low pressure CRSS sets with seismic anisotropy observations suggests that the CRSS data used in the present study are representative of deformation in the upper $200-250 \mathrm{~km}$ of the mantle (Mainprice et al., 2005). 
Table 1: Slip systems parameters used in the VPSC simulations*

\begin{tabular}{|c|c|c|}
\hline Slip Systems & Critical Resolved Shear Stress\# & Stress exponent \\
\hline$(010)[100]$ & 1 & 3 \\
\hline$(001)[100]$ & 1.5 & 3 \\
\hline$(010)[001]$ & 2 & 3 \\
\hline$(100)[001]$ & 3 & 3 \\
\hline$(011)[100]$ & 4 & 3 \\
\hline$(110)[001]$ & 6 & 3 \\
\hline$\{111\}\langle 110\rangle^{a}$ & $\beta$ & 3 \\
\hline$\{111\}<011>^{\mathrm{a}}$ & $\beta$ & 3 \\
\hline$\{111\}<101>^{\mathrm{a}}$ & $\beta$ & 3 \\
\hline
\end{tabular}

The shear strain rate in each slip system is proportional to the resolved shear stress on the slip plane in the slip direction normalized to the CRSS of the slip system raised to the power $n$. The iron-rich olivine polycrystals display a stress exponent $n$ of 4.1 , whereas typical mantle olivine has lower $n$ values (e.g., 3.5, Bai et al., 1991). We tested $n$ equal to 3 or 4 and observed little difference in terms of magnitude of anisotropy (cf. supplementary material, Fig. S1). In the following we focus on the results for $n=3$ in order to agree with most laboratory and previous numerical experiments on olivine polycrystals, which usually find stress exponent $n=3 \pm 0.5$.

A major challenge in modeling viscoplastic anisotropy of a rock (i.e., an aggregate of crystals with different orientations) is that traditional polycrystal plasticity models only account for deformation accom- modated by dislocation glide. Since olivine has fewer than 4 independent slip systems, deformation processes other than glide are required to ensure strain compatibility. To simulate the contribution of these other processes, Detrez et al. (2015) modified the SO-VPSC model by adding an isotropic deformation process. In the present study we build on this already validated development. To close the yield surface and simulate the contribution of isotropic deformation processes to plastic deformation, we add three fictitious pyramidal slip systems in the VPSC models: $\{111\}\langle 110\rangle,\{111\}\langle 011\rangle$ and $\{111\}\langle 101\rangle$, here referred as dummy slip systems. These slip systems were defined such that they may accommodate any imposed plastic strain, but do not contribute to plastic spin. They mimic therefore isotropic strain accommodation mechanisms, which do not contribute to CPO evolution. The contribution of the dummy systems to the total viscoplastic deformation is varied by changing their CRSS by a factor $\beta$ (Table 1). Thus, the present implementation differs from previous VPSC model applied to olivine polycrystals, referred thereafter as 'standard' VPSC model, since in the latter the dummy slip systems contributed to plastic spin and were not allowed to accommodate $>5 \%$ (therefore contributing only in a minor way to the CPO evolution) by using $\beta$ values $\geq 50$ (e.g., Wenk et al., 1991; Tommasi et al., 2000; Castelnau et al., 2009). 
Hardening is not considered in the present models since in most high-temperature ( $1200{ }^{\circ} \mathrm{C}$ ) deformation experiments on olivine aggregates, in the absence of significant microstructural changes, steady-state mechanical behavior is achieved after relatively small strains (e.g., Chopra and Paterson, 1984; Hansen et al., 2012b; Thieme et al., 2018). In the present models, diffusive processes are therefore implicitly ac- counted for in two ways: via their contribution to avoid hardening by dislocation entanglements and via the activation of the dummy slip systems. Indeed, although we do not model any specific additional process, but solely their contribution to strain accommodation in the olivine polycrystal, most possible additional processes, such as recrystallization, grain boundary migration or sliding, involve diffusion.

\subsection{Model setup}

Two series of tests were run to simulate the stresses measured in the laboratory: (1) simple shear parallel to a previous shearing deformation that produced the olivine $\mathrm{CPO}$, that is, shearing in the $\mathrm{X}$ direction and in a plane normal to the $\mathrm{Z}$ direction; (2) axial extension normal to the previous shear plane, parallel to the $\mathrm{Z}$ direction (Fig. 1). Lower bound simulations were run under kinematic boundary conditions by im- posing all components of the velocity gradient tensor $\mathrm{L}\left(\mathrm{s}^{-1}\right)$. For easy comparison between simple shear (ss) and axial extension (ext) simulations, $\mathrm{L}$ is defined with the condition that the norm of the strain rate tensor in both cases equals 1 (cf. supplementary material):

$$
L_{s s}=\left[\begin{array}{ccc}
0 & 1.4 & 0 \\
0 & 0 & 0 \\
0 & 0 & 0
\end{array}\right] ; L_{\text {ext }}=\left[\begin{array}{ccc}
0.4 & 0 & 0 \\
0 & 0.8 & 0 \\
0 & 0 & 0.4
\end{array}\right] ;
$$

In the VPSC simulations, mixed boundary conditions were enforced because they better mimic the actual conditions in laboratory experiments: imposed torsion on the plane normal to the piston axis or imposed axial extension and homogeneous stress in the plane normal to the piston axis.

$$
\begin{aligned}
& L_{s s}=\left[\begin{array}{ccc}
* & 1.4 & 0 \\
0 & 0 & 0 \\
0 & 0 & *
\end{array}\right] ; L_{\text {ext }}=\left[\begin{array}{ccc}
* & 0 & 0 \\
0 & 0.8 & 0 \\
0 & 0 & *
\end{array}\right] ; \text { ssett }=\left[\begin{array}{ccc}
0.5 & * & * \\
* & * & * \\
* & * & 0.5
\end{array}\right] ; \\
& \text { *=not imposed }
\end{aligned}
$$

We also ran VPSC simulations using fully kinematic boundary conditions. Differences between stresses predicted by TGT or SO-VPSC simulations using either kinematic or mixed boundary conditions are low $(<10 \%$, cf. supplementary material, Fig. S2), by consequence we focus on the results for mixed boundary conditions.

The models are run for a single deformation step of $1 \%$ Von Mises equivalent strain and the mechanical behavior is analyzed before actualization of the texture, in other words, we calculated the instantaneous mechanical response of the olivine polycrystals. The stresses predicted by the viscoplastic models in simple shear and in axial extension were compared to final stresses measured in the torsion experiments and to peak stresses in the extensional ones, respectively (Fig. 1). For internal consistency, stresses measured in the laboratory were normalized for a homogeneous Von Mises equivalent strain rate of $2 \times 10^{-4} \mathrm{~s}^{-1}$ (Table 2; the normalization procedure is presented in the Supplementary Material). Stresses calculated in the models are normalized by the strength of the easiest slip system, (010)[100]. In addition, for comparing the viscoplastic anisotropy observed in the laboratory to the one predicted by the different numerical models, Von Mises equivalent stresses for simple shear and axial extension were normalized by the Von Mises equivalent stress of the isotropic aggregate in each data set (experiments or different modeling approaches). 
Table 2: Model predictions and laboratory measurements

\begin{tabular}{|c|c|c|c|c|c|c|c|c|c|c|}
\hline \multicolumn{6}{|c|}{$\begin{array}{l}\text { Instantaneous stresses predicted by the models and final (simple shear) } \\
\text { and peak (extension) stresses measured in the experiments }\end{array}$} & \multicolumn{5}{|c|}{$\begin{array}{l}\text { Instantaneous stresses* predicted by } \\
\text { SO-VPSC models for different } \beta \text { values }\end{array}$} \\
\hline $\begin{array}{l}\text { Mechanical } \\
\text { Solicitation }\end{array}$ & $\begin{array}{l}\text { Initial } \\
\text { texture }\end{array}$ & $\begin{array}{c}S O- \\
V P S C^{*} \\
\beta=50\end{array}$ & $\begin{array}{c}\text { TGT- } \\
V P S C \\
\beta=50\end{array}$ & $\begin{array}{c}\text { Lower } \\
\text { Bound* }\end{array}$ & $\begin{array}{c}\text { Laboratory } \\
\text { Data } \\
(M P a)\end{array}$ & $\beta=10$ & 5 & 3 & 2 & 1 \\
\hline \multirow{4}{*}{$\begin{array}{l}\text { Simple } \\
\text { Shear }\end{array}$} & UNTEXTURED & 16.77 & 9.64 & 3.94 & - & 8.33 & 6.03 & 4.56 & 3.53 & 2.10 \\
\hline & PT0655 & 14.86 & 8.57 & 3.59 & 148.23 & 7.54 & 5.56 & 4.29 & 3.39 & 2.09 \\
\hline & PT0640 & 8.43 & 5.87 & 2.86 & 118.47 & 4.82 & 3.82 & 3.18 & 2.73 & 1.95 \\
\hline & PT0651 & 7.58 & 5.19 & 2.57 & 109.43 & 4.38 & 3.48 & 2.92 & 2.50 & 1.78 \\
\hline \multirow{4}{*}{ Extension } & UNTEXTURED & 16.72 & 10.05 & 3.89 & 168.00 & 8.27 & 5.97 & 4.52 & 3.50 & 2.08 \\
\hline & PT0655 & 18.00 & 11.20 & 3.94 & 201.24 & 8.64 & 6.12 & 4.56 & 3.50 & 2.06 \\
\hline & PT0640 & 28.04 & 20.82 & 6.00 & 199.44 & 12.31 & 8.03 & 5.53 & 3.98 & 2.14 \\
\hline & PT0651 & 37.13 & 33.32 & 5.69 & 209.85 & 14.88 & 9.08 & 6.00 & 4.22 & 2.23 \\
\hline
\end{tabular}

* Von Mises equivalent stress normalized by the strength of the (010)[100] slip system

\# Von Mises equivalent stress recalculated for a homogeneous strain rate of $2 \times 10^{-4} \mathrm{~s}^{-1}$; extension values are average ones.

The effect of the texture on the viscoplastic mechanical behavior was tested using four different initial CPOs: an untextured and three olivine CPOs measured at the end of the torsional tests by Hansen et al. (2012a), performed at high temperature and moderate pressure conditions (Fig. 1). These initial textures are representative of common $\mathrm{CPO}$ symmetries observed in natural and experimental samples deformed in simple shear and cover the entire range of $\mathrm{CPO}$ intensities measured in peridotite massifs and mantle xenoliths (Tommasi and Vauchez, 2015). In the models, grains are initially spherical. The untextured polycrystal is made of 1000 grains. The CPO intensity is characterized by the J-index, which is the integral of the squared orientation distribution function (ODF), quantified using the MTEX toolbox based on an ODF calculated using a "de la Vallée Poussin" kernel with a half width of $10^{\circ}$ (Mainprice et al., 2014). Sample PT0655, which was initially deformed to a shear strain of 1 , is represented by a texture composed of 870 grains. This texture is very weak ( $\mathrm{J}$-index of 1.5); it is characterized by a subtle double maximum of [100] at low and high angles to the shear direction and by a dispersed girdle of [010] at high angle to the maximum finite elongation with a weak maximum normal to the foliation. Samples PT0640 and PT0651, which underwent initial shear strains of 4 and 10, are represented by textures composed of 489 and 665 grains, respectively. Both have very strong olivine CPOs (J-index of 14.5 and 20.5, respec- tively) and are characterized by alignment of the [100] maximum with the shear direction and of the [010] maximum normal to the shear plane. However, the textures differ by the distribution of the [010] axes, which forms a girdle normal to the shear direction (X) in PT640 and a point maximum normal to the shear plane (XY plane) in PT655 (Fig. 1).

To verify if the modified SO-VPSC approach used in this work is capable of reproducing the $\mathrm{CPO}$ evolution recorded in simple shear deformation, we run simple shear tests up to shear strains of 20 for an initially untextured olivine polycrystal composed of 1000 grains. The simulations were conducted applying the same slip system parameters and (mixed) boundary conditions used to model the viscoplastic mechanical behavior. This study focuses on the SO-VPSC predictions because they have been shown to better reproduce the solutions of full-field simulations (Castelnau et al., 2008). The predictions of the TGT- VPSC 
model are nevertheless presented for continuity with previous work (e.g., Wenk et al., 2009; Tommasi et al., 1999, 2000).

\section{Results}

\subsection{Mechanical behavior}

Fig. 2a presents a comparison between the stresses normalized to the strength of the untextured polycrystal predicted by the different viscoplastic models (colored markers) at standard conditions (CRSS of the dummy slip systems in TGT and SO-VPSC models, $\beta=50$ ) and the experimental measurements (black circles), as a function of the intensity of the initial CPO. Both laboratory and numerical experiments show that textured olivine polycrystals deformed in simple shear parallel to the preexisting fabric (empty markers) are weaker than the untextured aggregate, whereas those deformed in axial extension normal to the preexisting fabric (filled markers) are harder. There is a positive correlation between CPO intensity and magnitude of viscoplastic anisotropy, defined as the ratio between the Von Mises equivalent stresses in extension and in simple shear tests, but the relationship is not linear

(Fig.

2b).

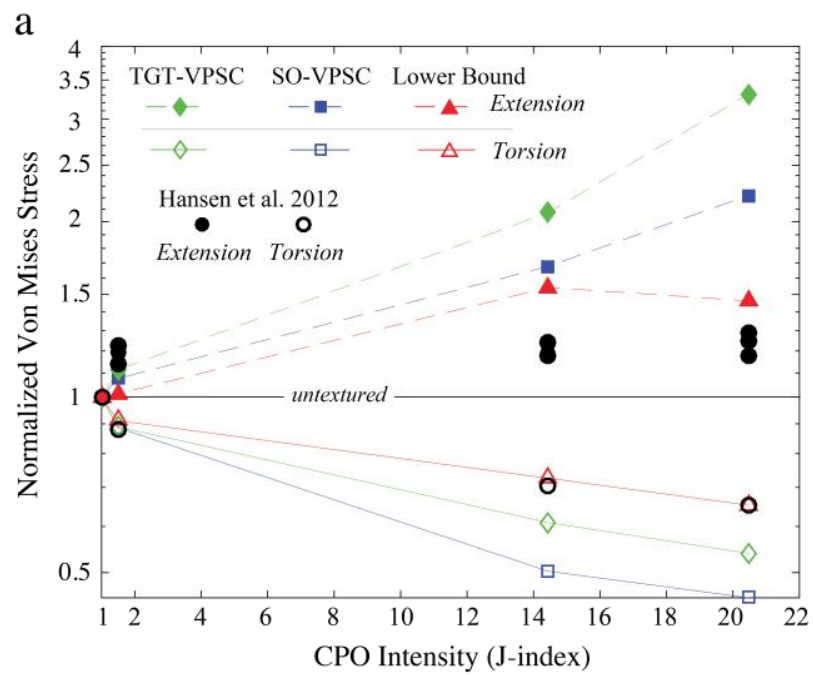

b

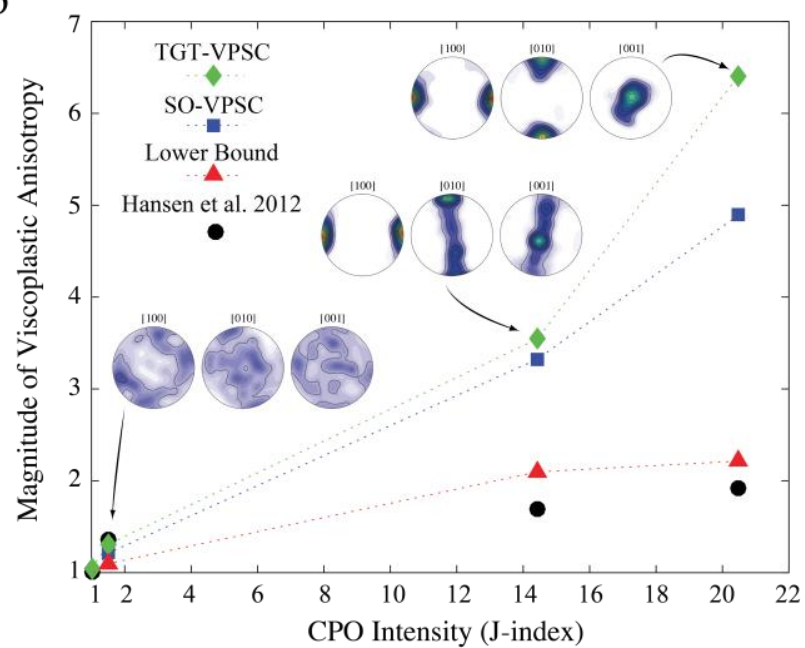

Fig. 2. (a) Instantaneous normalized Von Mises equivalent stresses (relative to behavior of an isotropic polycrystal) and (b) magnitude of viscoplastic anisotropy (ratio between Von Mises equivalent stresses in extension and simple shear) for lower bound and VPSC simulations (colored symbols), in which the CRSS of the dummy slip systems ( $\beta$ ) is set to 50 for the three initial olivine CPO (recalled in Fig. $2 \mathrm{~b}$ and presented as a function of the CPO intensity - J-index). Black circles indicate stresses measured in the laboratory experiments. The empty markers present the results for simple shear parallel to the [100] maximum in a plane normal to the [010] maximum of the initial CPO. The filled markers present the results for axial extension parallel to the [010] maximum of the initial CPO. For calculating the magnitude of anisotropy the stresses in extension were averaged; for the color legend and labels of the pole figures, as well as loading geometries, refer to Fig. 1.

In simple shear, the lower bound and TGT-VPSC models predict normalized stresses in reasonable agreement with the experimental data, but SO-VPSC simulations predict lower normalized stresses. In axial extension, the models overestimate the strength of the olivine polycrystals relative to the experimental measurements, except for the sample PT0655, which despite a very weak CPO, displays a stronger anisotropy than the one predicted by the models. The predictions of the lower bound approach are the closest to the experimental data and the TGT-VPSC models are the farthest. The discrepancy between numerical and laboratory experiments, which increases with increasing intensity of the $\mathrm{CPO}$, results therefore from overprediction of the strength of olivine polycrystals deformed numerically in extension. In this loading geometry, most crystals are in hard orientations relative to extension axis, that is, low resolved shear stresses are expected on the three main slip systems of olivine: 
(010)[100], (001)[100] and (010)[001]. By consequence, high stresses are required to deform the polycrystal by dislocation creep.

The lower bound model, which only enforces stress equilibrium and not strain compatibility, predicts the lowest polycrystal strengths for all textures and loading geometries. The better fit of the experimental data by the lower bound approach points to the major role of enforcing strain compatibility in modeling the mechanical behavior of olivine polycrystals. Further analysis of the stresses predicted by the different models (Fig. 3; Table 2) corroborates this point. The SO-VPSC, which most strictly enforces strain compatibility and better fits fullfield polycrystal plasticity simulations (Castelnau et al., 2006), predicts the highest stress values for both simple shear and extension.

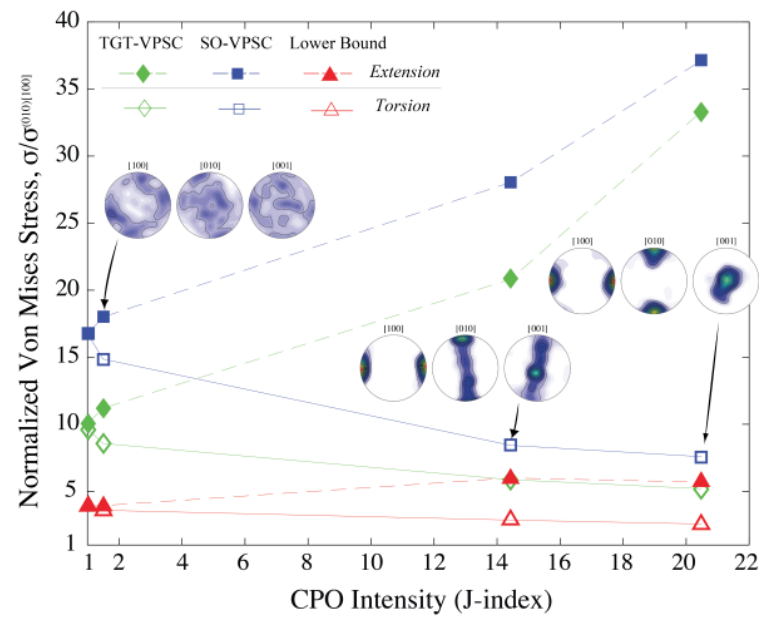

Fig. 3. Instantaneous Von Mises equivalent stresses (normalized to the strength of the easy (010)[100] slip system) predicted by different polycrystal plasticity models as a function of the initial CPO intensity for simple shear (empty markers) and axial extension (filled markers). The strength of the dummy slip systems $(\beta)$ is set to 50 . Pole figures show the CPO measured at the end of the torsion experiments (Hansen et al., 2012a), which are used as the initial CPO in the models; for the color legend and labels of the pole figures, as well as loading geometries, refer to Fig. 1.

The high stresses predicted in axial extension by the SO-VPSC model for the strongly textured polycrystals PT0640 and PT0651 result from low activities of the (010)[100] and (001)[100] slip systems, which have the lowest CRSSs (Fig. 4). For these textures, resolved shear stresses on these two slip systems are low in most crystals (i.e., extension is applied parallel to the maximum concentration of [010] axes and normal to the maximum concentrations of [100] and [001] axes). To accommodate the imposed extensional deformation, the very hard $(\beta=50)$ dummy slip systems have to be activated in many crystals. Even though these systems contribute to $<5 \%$ of the total deformation (Fig. 4), this moderate amount of activation still requires high stresses. The (010)[001] slip system, which has intermediate CRSS values, contributes significantly to the total deformation $(\sim 55 \%)$ in axial extension. In most crystals, resolved shear stresses on the (010)[001] slip system are also low, but the high stresses attained in the extensional deformation allow their activation.

In contrast, by imposing simple shear parallel to the maximum concentration of [100] olivine axes in a plane parallel to the maximum concentration of (010) planes in the strongly textured polycrystals PT0640 and PT0651, we obtain maximum resolved shear stresses on the easy slip system (010)[100], which accommodates $>50 \%$ of the deformation. In both simple shear and axial extension, the activity of the (010)[100] slip system is higher in the more strongly textured polycrystal (PT0651) than in the polycrystal with a CPO of intermediate strength. A closer analysis of the slip system activities highlights nevertheless some subtle differences between predictions for samples PT0640 and PT0651 (Fig. 4). This variation may be explained by differences in the symmetry of the CPO between the two polycrystals. PT0640 (J-index of 14.1) has a girdle distribution of [010] and [001], which allows in axial extension for high resolved shear stresses on (010)[001] in a larger number of crystals relative to PT0651 (J-index of 20), which has point distributions of the three main axes. 


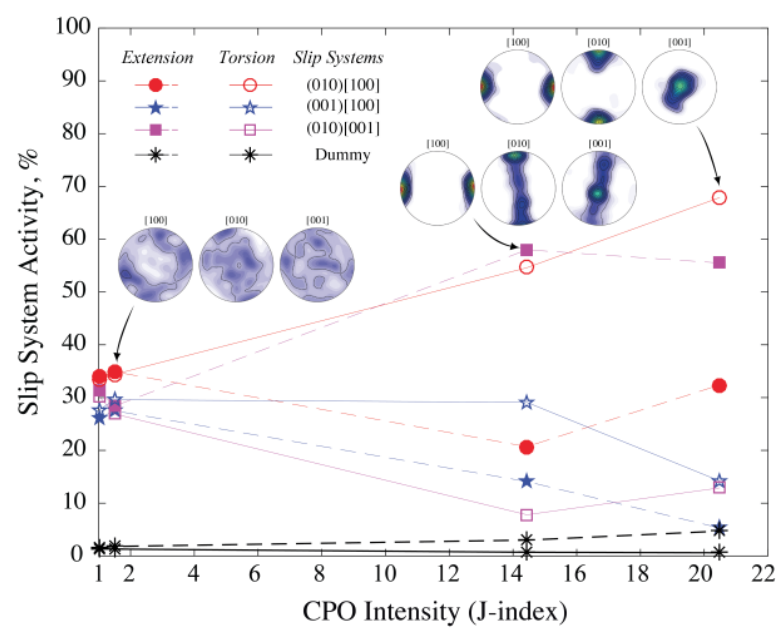

Fig. 4. Slip system activity predicted by the SO-VPSC model for simple shear (empty markers) and axial extension (filled markers). The sum of the activity of the three dummy systems, that is, the total contribution of the strain accommodation processes, is shown as black asterisks. Strength of the dummy slip systems $(\beta)$ is set to 50 in these simulations. The activity of (100)[001], (011) [100], and (110)[100] slips systems, which accommodate less than $1 \%$ of viscoplastic deformation, is not represented. Pole figures show the CPO measured at the end of the torsion experiments (Hansen et al., 2012a), for which the instantaneous viscoplastic response are calculated; for the color legend and labels of the pole figures, as well as loading geometries, refer to Fig. 1.

The observed discrepancy between laboratory results and model predictions advocates in favor of a significant contribution of others processes in addition to dislocation glide during deformation of olivine aggregates at laboratory conditions. This contribution appears to be particularly important if most crystals in the aggregate are unfavorably oriented to deform by dislocation glide on the easy slip systems, such as the present extensional tests on the strongly textured polycrystals. To evaluate if an increase of the contribution of isotropic deformation processes allows reproducing the stresses measured experimentally, the CRSS of the dummy slip systems was systematically decreased. The results are presented in the following section.

\subsection{Strain accommodation by additional isotropic deformation processes}

We tested the effect of increasing the contribution of isotropic deformation processes on the aggregate strength by decreasing the
CRSS of the dummy slip systems $(\beta=10,5,3$, $2,1)$ in the SO-VPSC simulations. These dummy systems represent any additional process that allows olivine crystals to deform even if oriented in a manner that results in very low resolved shear stresses on the three main slip systems: (010)[100], (001)[100], and (010)[001]. The stress predictions in simple shear and axial extension as a function of CPO intensity for different $\beta$ values are shown in Fig. 5 and in Table 2.
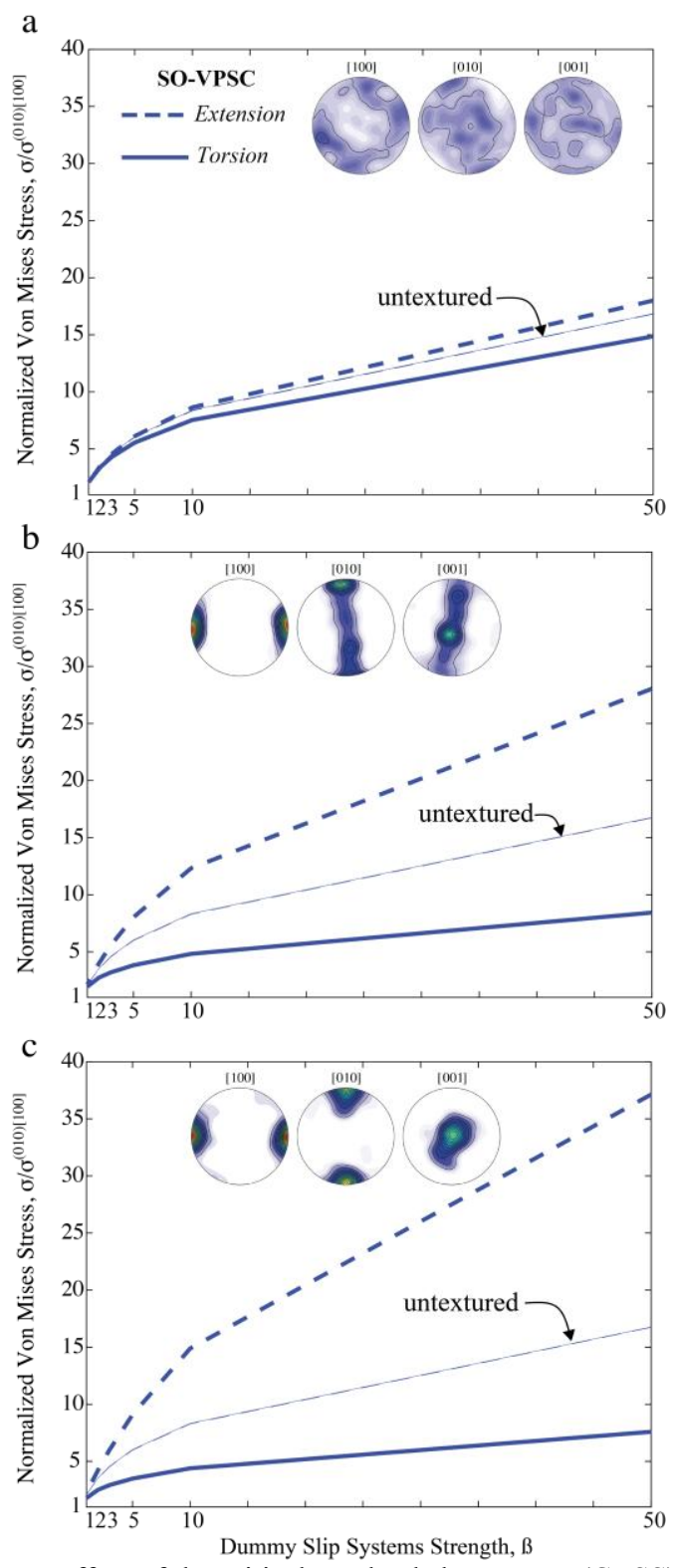

Fig. 5. Effect of the critical resolved shear stress (CRSS) of the dummy slip systems $(\beta)$ on the Von Mises equivalent stress (normalized to the strength of the easy (010)[100] slip system) predicted by the SO-VPSC model for simple 
shear (thick solid lines) and axial extension (thick dashed lines) using the three CPOs: (a) PT0655, (b) PT0640, and (c) PT0651, created in the torsion experiments (Hansen et al., 2012a). For reference, the mechanical behavior of an untextured polycrystal (isotropic) is displayed by a thin dashed (axial extension) or solid (simple shear) lines in all three figures; for the color legend of the pole figures and loading geometries, refer to Fig. 1.

A decrease in $\beta$ results in a non-linear decrease in the polycrystal strength for all textures and both loading geometries (Fig. 5ac). The rate of decrease varies as a function of CPO intensity and of loading geometry. The weakly textured aggregate deforms at similar stresses in both loading geometries (i.e., very weak anisotropic behavior) even at high values of $\beta$. In contrast, for the strongly textured aggregate (Fig. 5b,c), decreasing $\beta$ leads to distinct behaviors for the two loading

a

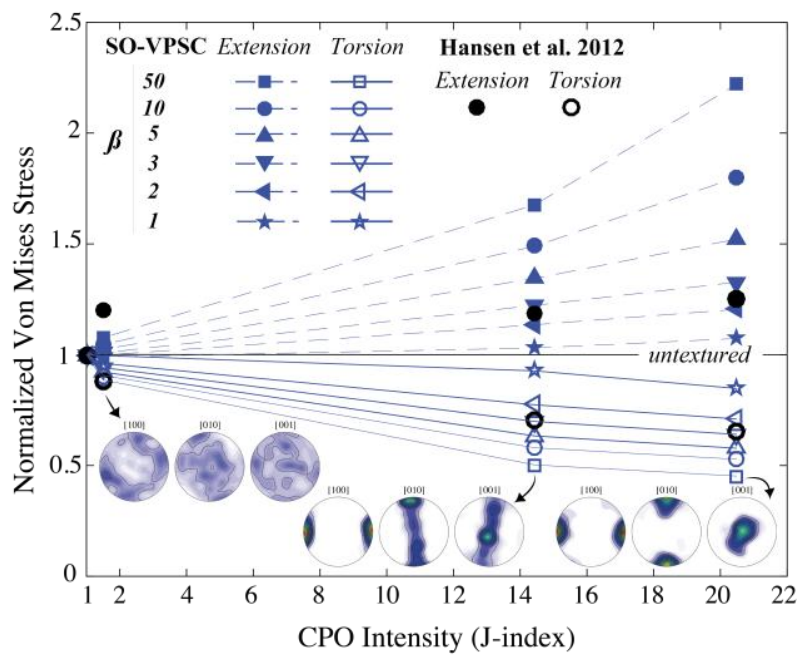

geometries: the reduction in the polycrystal strength is more marked in the axial extension test than in the simple shear simulations. It results therefore in reduction of the magnitude of anisotropy with decreasing $\beta$ (Fig. 6a). The modified SO-VPSC simulation in which the dummy systems (that allow for isotropic deformation) are 3 times harder to activate than the (010)[100] and (001)[100] easy slip systems $(\beta=3)$ better reproduces the mechanical behavior measured in the laboratory; except for the sample with the weakest CPO (PT0655), for which all models underestimate the magnitude of anisotropy (Fig. 6a,b). Differences in mechanical predictions between TGT- and SOVPSC models are only observed for $\beta>10$ (cf. supplementary material, Fig. S3).

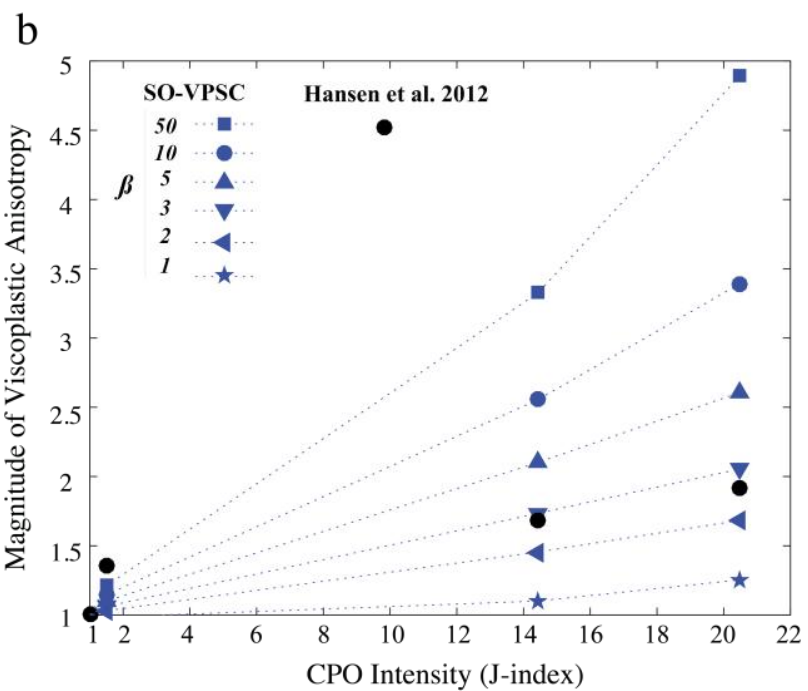

Fig. 6. a) Instantaneous Von Mises equivalent stresses normalized by the behavior of an isotropic polycrystal and (b) magnitude of the viscoplastic anisotropy predicted by SO-VPSC simulations with CRSS of the dummy systems ( $\beta$ ) between 50 and 1 for the three experimental olivine CPO as a function of increasing CPO intensity (J-index). Empty markers show results for simple shear parallel to the [100] maximum in a plane normal to the [010] maximum of the preexisting CPO and filled markers indicate the results for axial extension parallel to the [010] maximum of the preexisting CPO. The experimental data is represented by the black circles; for the color legend of the pole figures and loading geometries, refer to Fig. 1.

The change in mechanical behavior with decreasing $\beta$ may be explained by variations in the relative activities of the actual olivine slip systems and of the dummy ones, which represent additional deformation processes activated to ensure strain compatibility constraints (Fig. 7). In the VPSC model with $\beta=50$, the three main slip systems (010)[100], (001)[100], and (010)[001] account for at least $95 \%$ of the viscoplastic deformation in axial extension and
$99 \%$ of the deformation in simple shear. As $\beta$ decreases, the activity of the dummy slip systems increases at the expense of the activity of the actual ones. In a untextured aggregate (Fig. 7a), reduction of $\beta$ from 50 to 10 results in an increase of the activity of the dummy slip systems from $<1 \%$ to $\sim 11 \%$, for both simple shear and axial extension. For $\beta=5$, their activity increases to $\sim 30 \%$. A further decrease in 
$\beta$ to 3 or 2 , which is the range of values that better agrees with the mechanical data measured in the laboratory, leads to activities of the dummy slip systems of $\sim 45 \%$ or $\sim 60 \%$, respectively. For $\beta=1,>80 \%$ of the deformation is accommodated by the dummy slip systems. These high activities of the relaxation mechanism, which is represented in the present study by the dummy slip systems, are similar to those previously observed by Detrez et al. (2015).

The presence of an olivine $\mathrm{CPO}$ results in different relative slip system activities in axial extension and simple shear (Fig. 7b-d). This difference increases with increasing $\mathrm{CPO}$ intensity, because in a strongly textured aggregate, a larger proportion of the crystals will be either well or poorly oriented to
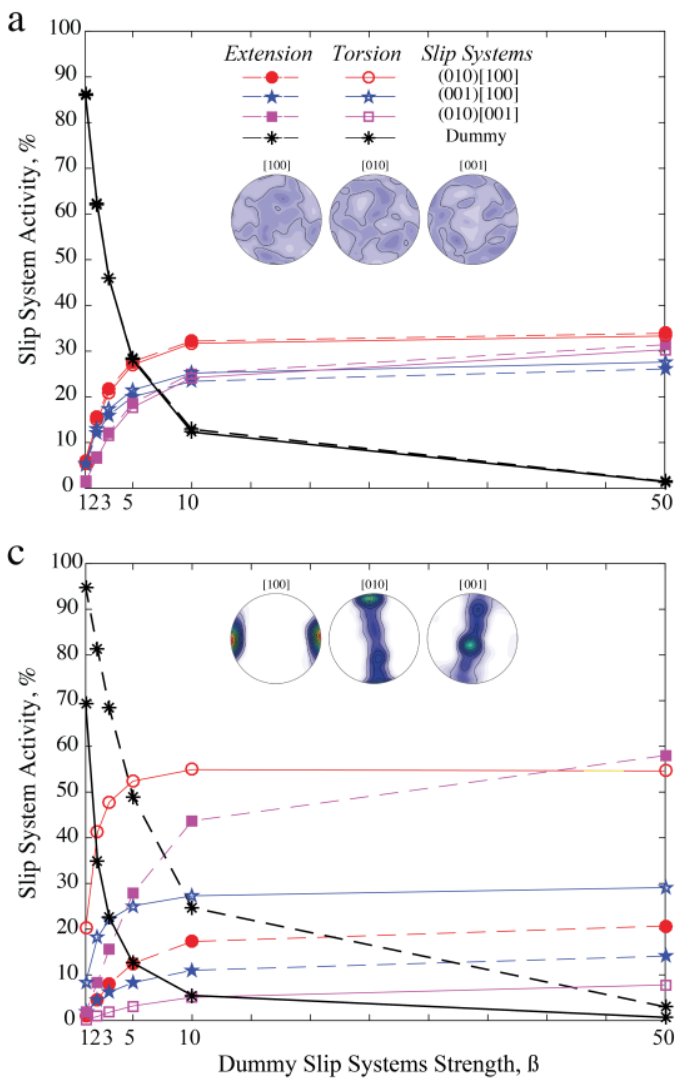

accommodate the imposed deformation. The three olivine aggregates used in the present simulations have textures in which most crystals are well oriented to accommodate the imposed simple shear, but poorly oriented to accommodate the imposed axial extension. For $\beta$ equal to 5 or 3 , the isotropic mechanism in simple shear respectively accommodates $\sim 25 \%$ or $\sim 40 \%$ (PT0655), $\sim 15 \%$ or $\sim 25 \%$ (PT0640), and $\sim 10 \%$ or $\sim 20 \%$ (PT0651) of the total viscoplastic deformation, whereas in axial extension it accommodates $\sim 30 \%$ or $\sim 50 \%$ (PT0655), $\sim 50 \%$ or $\sim 70 \%$ (PT0640), and $\sim 60 \%$ or $\sim 75 \%$ (PT0651), (Fig. $7 \mathrm{~b}-\mathrm{d}$ ). b

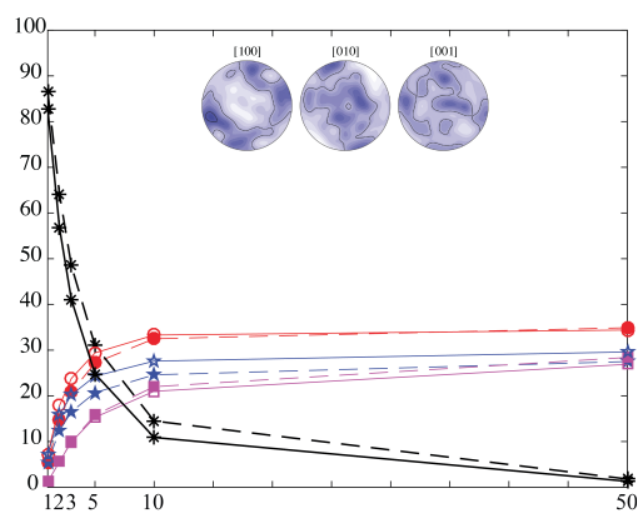

d

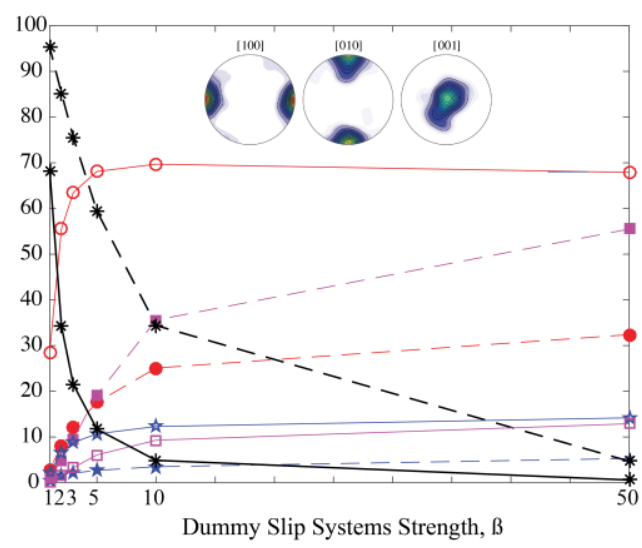

Fig. 7. Effect of the dummy slip system strength $(\beta)$ on the activity of the slip systems predicted by SO-VPSC models for the polycrystals: (a) untextured, (b) PT0655, (c) PT0640, and (d) PT0651. Empty markers represent simple shear simulations and filled markers axial extension simulations. The activity of the (100)[001], (011)[100], and (110)[100] slips systems, which accommodate less than $1 \%$ of viscoplastic deformation, is not shown. The sum of the activity of the three dummy systems, that is, the total contribution of the strain accommodation processes, is shown as black asterisks; for the color legend of the pole figures and loading geometries, refer to Fig. 1.

It is noteworthy that for $\beta \leq 5$, the dummy slip systems remain active even if most crystals in the aggregates are well oriented to deform by dislocation glide on the easy 
(010)[100] and (010)[001] slip systems, such as when simple shear is applied on the strongly textured aggregates (PT0640 and PT0651). Their isotropic character may explain this behavior; there is always at least one system among the 12 systems that compose the three dummy slip modes on which the resolved shear stresses are high.

\subsection{Olivine CPO evolution}

To test if the modified SO-VPSC model that better fits the viscoplastic anisotropy measured in the experiments can also reproduce the texture evolution in simple shear, we compare the CPO evolution predicted in simulations with different CRSSs for the dummy slip systems $(\beta=50,5$, or 3$)$ to $\mathrm{CPO}$ data for olivine polycrystals deformed experimentally in simple shear at high temperature conditions (Zhang and Karato, 1995; Bystricky et al., 2000; Demouchy et al., 2012; Hansen et al., 2012a,b,c, 2014). Fig. 8 displays the evolution of the CPO intensity characterized by the J-index and the angle between the maximum eigenvector of the [100] axis distribution and the shear direction as a function of the shear strain. Note that the CPO evolution of the olivine aggregates presented by Hansen et al. (2012a), for which the mechanical responses were simulated in the present study, is also included in Fig. 8. In this figure, we also compare the CPO evolution predicted by the present modified SO-VPSC model to those of a
VPSC model modified to account for dynamic recrystallization by subgrain rotation in olivine aggregates (CDRX-TG-VPSC; for details on the model cf. Signorelli and Tommasi, 2015). The CRSSs in this previous model are slightly different from those used in the present study, but the relative strengths between slip systems are maintained. Thus, we do not expect significant differences in CPO evolution. Pole figures displaying the evolution of the olivine CPO in the SO-VPSC model with $\beta=50,5$, or 3 are shown in the Supplementary material (Fig. S5).

The SO-VPSC simulations in which the strength of the dummy slip systems $(\beta)$ is equal to 50 show a rotation of the [100] axes towards the shear direction that closely follows the rotation of the finite strain ellipsoid, poorly reproducing the experimental data. A decrease in $\beta$ leads to faster rotation of [100] maximum towards the shear direction, which better reproduces the CPO reorientation trend observed in the experiments. However, for low strengths of the dummy slip systems $(\beta \leq 3)$, that is, when the additional deformation process are very active, and at high shear strains $(\geq 5)$, the CPO evolution departs from a continuous trend: the maximum concentration of [100] oscillates between $0^{\circ}$ and $10^{\circ}$ from the shear direction (Fig. 8). Models with $\beta=5$ also exhibit a similar behavior, but the oscillations start at higher shear strains $\quad(\sim 10)$ and are smaller $\left(\geq 5^{\circ}\right)$.
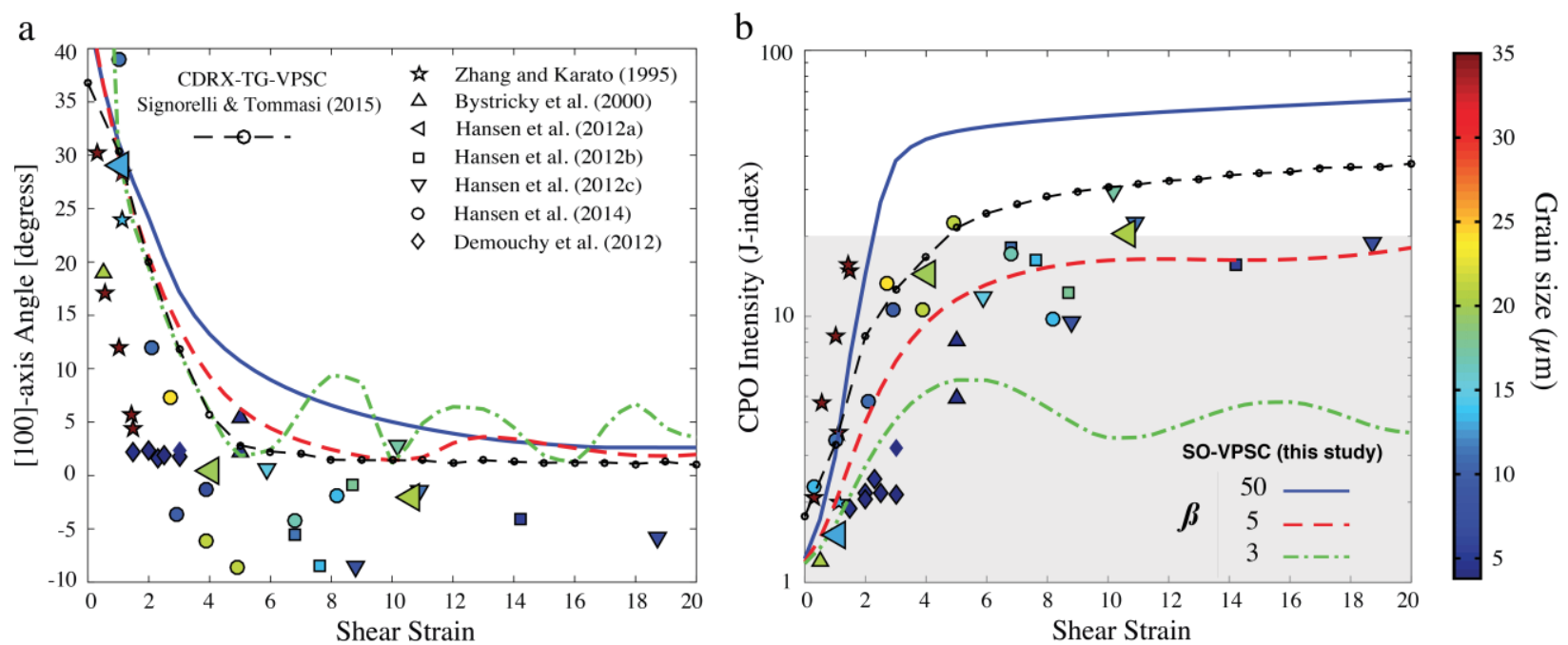

Fig. 8. CPO evolution as a function of increasing shear strain predicted by SO-VPSC simulations with different dummy slip 
system strengths $(\beta)$. a) CPO orientation defined by the angle between shear direction and the maximum eigenvector of the [100]axis orientation distribution. b) CPO intensity evolution characterized by the J-index. Experimental data from Zhang and Karato (1995), Bystricky et al. (2000), Demouchy et al. (2012), and Hansen et al. (2012a,b,c,2014) are displayed for comparison. The CPO evolution of the olivine aggregates in Hansen et al. (2012a), in which the viscoplastic response were simulated in the present study, is highlighted as larger left-pointing triangles. The color gradient in the experimental data symbols indicate the average grain sizes. The predictions of the CDRX-TG- VPSC model by Signorelli and Tommasi (2015), which simulates recrystallization by subgrain rotation, are shown by the dotted-dashed line. The pale gray rectangle in (b) indicates the range of J-index measured in > 600 natural samples (Tommasi and Vauchez, 2015). (For interpretation of the references to colour in this figure legend, the reader is referred to the web version of this article).

Analysis of the evolution of the CPO intensity shows that the decrease in the strength of the dummy slip systems results in stabilization of the CPO at lower J-index values (Fig. 8b). This observation highlights the essential role of additional processes, such as diffusion or dynamic recrystallization, on the evolution of the CPO intensity. CPO intensities measured in the high shear strain experiments are significantly overestimated for VPSC simulations with $\beta=50$ and underestimated for VPSC simulations with $\beta=3$, but they are well reproduced by models with $\beta=5$. As for the $\mathrm{CPO}$ orientation, periodic oscillation of the $\mathrm{CPO}$ intensity is observed for shear strains $\geq 5$ for $\beta=$ 3 (Fig. 8). In Fig. 8, the experimental data is colored as a function of the average grain size. CPO intensity saturates at lower values in the finer grain size aggregates, being better fitted by models with low $\beta$ values. This observation provides indication that the additional deformation process might be grain-size sensitive.

In conclusion, the modified SO-VPSC model with $\beta=5$ better reproduces the $\mathrm{CPO}$ evolution in simple shear or torsion experiments than standard SO-VPSC simulations in which most of the deformation is accommodated by dislocation glide on the actual olivine slip systems $(\beta=50)$. CPO evolution in the modified SO-VSPC model with $\beta=5$ is similar, though less stable at shear strains $\geq 10$, to the one predicted by the VPSC model modified to account for dynamic recrystallization by subgrain rotation (CDRX-TG-VPSC model; Signorelli and Tommasi, 2015). The modified SO-VPSC model with $\beta=3$, which better reproduces the viscoplastic anisotropy measured in the experiments, predicts well the evolution of the CPO orientation observed in simple shear or torsion experiments up to shear strains of $\sim 5$, but it underestimates the CPO strength and diverges from the experimental data at higher shear strains.

\section{Discussion}

\subsection{Viscoplastic anisotropy under laboratory conditions}

All polycrystal plasticity models analyzed in the present study reproduce qualitatively the mechanical results of Hansen et al. (2012a): simple shear in a direction parallel to the [100] maximum on a plane normal to the [010] maximum requires lower stresses than axial extension parallel to the [010] maximum (Fig. 2a). This behavior may be explained by simple geometrical considerations. In simple shear, re- solved shear stresses on the easy slip systems, (010)[100] and (001) [100], are high for most olivine crystals composing the aggregate, whereas if axial extension is imposed parallel to the [010] maximum resolved shear stresses are very low on these easy slip systems for most crystals.

Despite the qualitatively coherent results, the polycrystal plasticity models overestimate the magnitude of viscoplastic anisotropy relative to laboratory experiments for polycrystals with a well-developed CPO (Fig. 2b). Based on the better agreement between the lower bound model predictions and the experimental data, we infer that the discrepancy between numerical models and experiments arises from imposing strain compatibility in a system that does not have enough degrees of freedom ( $<4$ independent slip systems). In the experiments, other strain accommodation mechanisms are active (recrystallization, grain boundary sliding or migration, diffusional mass transfer, climb, formation of kinks...). Some of 
these processes are isotropic, others are not. The fact that the viscous anisotropy measured in the laboratory is lower than the one predicted by VPSC simulations in which most of the deformation is accommodated by dislocation glide $(\beta=50)$ implies nevertheless that these additional processes play an important role in strain accommodation in the experiments. To test this hypothesis, we reduced the critical resolved shear stress of the dummy slip systems, which simulate the contribution of isotropic deformation processes to strain accommodation in the VPSC simulations. For strongly textured aggregates, models in which the strength of the dummy slip systems is 3 times higher than the strength of the easiest (010)[100] slip system in olivine do reproduce the magnitude of anisotropy measured in the laboratory experiments (Fig. 6). In these models, the dummy systems contribute more to the total deformation when the polycrystal is in hard orientation than when it is in a soft one (Fig. 7), decreasing the magnitude of anisotropy. The present results are consistent with the previous analysis by Hansen et al. (2016b), in which the mechanical data from the experiments simulated here together with other experiments on olivine aggregates were fitted by a pseudo-Taylor model that relaxed the strain compatibility constraint.

The SO-VPSC model modified to account for additional isotropic deformation processes, which produce plastic strain but no plastic spin, also better reproduces the CPO evolution measured in torsion experiments for $\beta$ $=5$ compared to predictions of the 'standard' VSPC model $(\beta=50)$. Higher contribution of the isotropic processes, which is associated with low $\beta$ values, results in faster rotation of [100] maximum relative to shear plane and in slower evolution of the CPO intensity (Fig. 8). These simulations reveal a CPO evolution in simple shear similar to the one predicted by the VPSC model modified to account for dynamic recrystallization by subgrain rotation, in which the strain compatibility was relaxed within subgrains (Signorelli and Tommasi, 2015). Both models therefore hint at the essential role of additional processes, which accommodate strain components that cannot be produced by dislocation glide in the deformation of olivine polycrystals. The modified VPSC model presented here therefore represents an acceptable alternative to predict olivine $\mathrm{CPO}$ evolution in simple shear, with a significantly lower calculation cost than VPSC models explicitly incorporating recrystallization.

The modified SO-VPSC simulations with $\beta=3$, which better fit the viscoplastic anisotropy measured in the experiments, predict correctly the texture evolution observed in simple shear experiments up to shear strains of 5 , but diverge from the experimental data by displaying an oscillatory behavior at higher shear strains. This oscillation is consistent with the fact that ellipsoidal inclusions embedded in a deformable media do not have any stable position in simple shear, but rotate continuously (Jeffery, 1922). Indeed, low $\beta$ values result in a low strength of the equivalent homogeneous medium that represents the polycrystal in VPSC. This interpretation is corroborated by the analysis of the SO-VPSC model in which the critical (maximum) aspect ratio of the crystals is changed; higher critical aspect ratios result in higher oscillations of the CPO orientation (cf. supplementary material, Fig. S4).

Comparison of the VPSC predictions to the experimental data suggests that although isotropic, the additional strain accommodation processes are associated with a non-linear mechanical behavior. The study of Detrez et al. (2015), which first introduced additional isotropic deformation processes into the VPSC model, showed that significant activation of a Newtonian isotropic process resulted in a significant decrease of the apparent stress exponent of the polycrystal relative to the stress exponent associated with dislocation creep. The present study suggests that the mechanical data of Hansen et al. (2012a) are better reproduced by simulations in which the isotropic mechanisms accommodate a substantial proportion of the total viscoplastic deformation $(\geq 20 \%$ in simple shear and $\geq 50 \%$ in axial extension). Such a significant contribution from the isotropic processes would surely decrease the stress exponent if these processes were Newtonian. Yet, stress exponents measured in torsional experiments at high finite strains on polycrystals 
comprised by olivine $\mathrm{Fo}_{50}(n=4.1$, Hansen et al., 2012b) and Fo90 ( $n=3.3$, Bystricky et al., 2000) are high. We therefore suggest that the additional strain accommodation processes are probably non- Newtonian.

\subsection{Viscoplastic anisotropy under upper mantle conditions}

In the present simulations, we do not model specific additional deformation processes, but analyze their effect on the viscoplastic mechanical behavior of the textured rock (polycrystal) by modeling their contribution to strain accommodation. In other words, we calculate how the operation of these processes reduces the geometrical hardening. However, to extrapolate the conclusions of our analysis to the upper mantle, assumptions must be made about the nature of these processes. Increased amounts of strain from diffusive mass transfer or sliding on grain boundaries relative to strain from dislocation glide are favored by smaller grain sizes. This grain-size dependence has been clearly imaged in situ by the difference in strain distribution in deformation experiments on halite aggregates with different grain sizes (Bourcier et al., 2013). Thus, these grain-size sensitive processes are potentially more active in experiments that use fine-grained olivine polycrystals $(5-20 \mu \mathrm{m})$ than during deformation of coarse-grained peridotites in the upper mantle. Indeed, strain rates measured during axial compression experiments on fine-grained $(<10$ $\mu \mathrm{m})$ olivine aggregates were up to 4.6 times higher than strain rates predicted by a lower bound model for the same textures and loading conditions, but no enhancement in strain rate relative to the models was observed for experiments on aggregates with coarser $(>100$ $\mu \mathrm{m})$ grain sizes (Tielke et al., 2016). In the present study, grain-size sensitivity of the additional processes is suggested by the comparison of the evolution of the CPO intensity in simple shear predicted by the modified SOVPSC model and the CPO intensity measured experimentally (Fig. 8b): the observations on the finest-grained aggregates are better fitted by models with lower $\beta$ values, in which the dummy slip systems representing these additional processes are more active. One should note that the present results do not imply that grain-size sensitive processes are isotropic these processes might be anisotropic in the presence of a shape-preferred orientation (Wheeler, 2010). The present work solely indicates that the additional processes operating in the experiments allow avoiding strain compatibility problems arising from the viscoplastic anisotropy of the olivine crystal by accommodating strain components that cannot be achieved by the activation of the easy slip systems in olivine.

The partitioning of deformation among different processes (dislocation glide, diffusive mass transfer, grain-boundary sliding, and others) depends on temperature, stress, and chemical environment, but all other conditions being similar, an increase in grain size should favor dislocation glide. Olivine grain sizes in naturally deformed upper mantle rocks are typically plurimillimetric. Finer-grained peridotites are limited to shear zones $(<1 \mathrm{~km}$ wide) localized in the shallow parts of the lithospheric mantle and, even within these shear zones, in the absence of synkinematic reactions, olivine grain sizes are most often $>100 \mu \mathrm{m}$ (cf. Tommasi and Vauchez, 2015; Vauchez et al., 2012). Thus, although strain rates and stresses are lower than in the experiments, one may expect a greater contribution of dislocation glide to plastic deformation in most of the upper mantle. If that is the case, our results suggest that the viscoplastic anisotropy of the upper mantle might be higher than the one measured in the experiments and better represented by the VPSC simulations in which the contribution of the dummy slip systems is reduced $(\beta>10)$.

By consequence, previous studies that coupled the standard VPSC model to finite element simulations, such as Knoll et al. (2009), Tommasi et al. (2009), and Blackman et al. (2017), have estimated the maximum effect of olivine viscoplastic anisotropy on the reactivation of preexisting plate-scale structures for creating new plate boundaries (Tommasi et al., 2009) and on the flow and seismic anisotropy patterns beneath oceanic plates (Blackman et al., 2017). On the other hand, the ratios of $10-100$ 
between normal and shear viscosity used in convection models by Christensen (1987), Lev and Hager (2008, 2011), and Becker and Kawakatsu (2011) moderately to strongly overestimate viscous anisotropy associated with strain-induced CPO in the upper mantle. Indeed, the CPO-induced viscoplastic anisotropy in olivine-rich rocks predicted by standard SOVPSC models does not surpass a factor 6 .

\section{Conclusion}

The viscoplastic anisotropy of olivine polycrystals deformed in simple shear and axial extension was modeled using three polycrystal plasticity models: the uniform stress (lower bound) approach and the tangent and secondorder VPSC models, which was modified to account for variable contribution of other deformation processes in addition to dislocation glide. The instantaneous mechanical responses predicted in the various simulations were compared to stresses measured in laboratory experiments for similar textures, loading geometries, and boundary conditions.

Laboratory data and numerical experiments suggest that the intensity of the viscoplastic anisotropy depends non-linearly on the CPO intensity; it increases faster for weak to moderate CPO intensities than at high CPO strengths. VPSC simulations in which strain is essentially accommodated by dislocation glide $(\beta=50)$ slightly underestimate the strength of the strongly textured aggregates relative to the strengths measured experimentally in torsion, but strongly overestimates the strength relatively to the experimental data in axial extension. This leads to overprediction of the viscoplastic anisotropy. Lower bound simulations, in which strain compatibility constraints are relaxed, better fit the experimental data. This suggest that in the laboratory experiments additional deformation processes operate, allowing the accommodation of the strain components that cannot be achieved by the activation of the easy slip systems in olivine.

The experimental data for the strongly textured aggregates is indeed well reproduced by the SO-VPSC model that includes dummy slip systems simulating additional isotropic strain accommodation processes without producing grain rotation. The best fit to the experimentally measured viscoplastic anisotropy is obtained for simulations in which the CRSS of the dummy systems is 3 times higher than the CRSS of the easiest slip system in olivine, (010)[100]. In these models, the dummy slip systems accommodate up to $50 \%$ of the viscoplastic deformation in axial extension and up to $20 \%$ of the deformation in simple shear. This modified SO-VPSC model also better reproduces the texture evolution measured in torsion experiments than the standard VPSC model. However, the best fit is obtained for simulations with lower activity of additional isotropic processes $(\beta=5)$; simulations with $\beta=3$ underestimate the CPO strength relatively to the experimental data and do not evolve into a stable orientation at high shear strains.

The present work implies that the viscoplastic anisotropy associated with deformation by dislocation creep in the upper mantle may be decreased by the operation of additional strain accommodation processes. If we assume that in most of the upper mantle the contribution of deformation processes such as grain boundary sliding or diffusive mass transfer is lower than in the experiments due to larger grain sizes, the viscoplastic anisotropy should be well predicted by the standard SO- VPSC model. In high-stress shear zones in the shallow part of the lithospheric mantle, in which grain sizes are small, the viscoplastic anisotropy is probably reduced and better predicted by the modified SO- VPSC model. The standard $(\beta=50)$ and modified ( $\beta=3-5)$ SO-VPSC models therefore reflect the possible range of texture-induced viscoplastic anisotropy in the upper mantle, that is, the viscous anisotropy relevant to domains of the upper mantle deforming dominantly by dislocation creep. The present simulations, which use CRSS for the olivine slip systems derived from high temperature low pressure single crystal experiments, provide good predictions of anisotropic viscous deformation for the upper $200-250 \mathrm{~km}$ of the mantle. Different CRSS derived from high-pressure experiments should be used for deeper parts of the upper mantle. 


\section{Acknowledgments}

We thank Ricardo Lebensohn and Carlos Tomé for making the code VPSC7c, which includes most of the last developments in viscoplastic self-consistent modeling, freely available. We also gratefully acknowledge Alain Vauchez, Sylvie Demouchy, Catherine Thoraval for discussions and Manuel Thieme and Sonia Ouadahi for comments on the figures. Anonymous reviewers are thanked for insightful comments. This project received funding from the European Union's Horizon 2020 research and innovation program under the Marie Sklodowska-Curie grant agreement No. 642029 (ITN-CREEP) and from the CNRS (France) and CONICET (Argentine) under the Project International de Cooperation Scienti que MicroTex (No. 067785).

\section{References}

Bai, Q., Mackwell, S.J., Kohlstedt, D.L., 1991. High-temperature creep of olivine single crystals 1 . Mechanical results for buffered samples. J. Geophys. Res. 96, 2441-2460.

https://doi.org/10.1029/90JB01723.

Becker, T.W., Kawakatsu, H., 2011. On the role of anisotropic viscosity for plate-scale flow. Geophys. Res. Lett. 38, 1-5. https://doi.org/10.1029/2011GL048584.

Blackman, D.K., Boyce, D.E., Castelnau, O., Dawson, P.R., Laske, G., 2017. Effects of crystal preferred orientation on upper-mantle flow near plate boundaries: rheologic feedbacks and seismic anisotropy. Geophys. J. Int. 210 (3), 1481-1493. https://doi. org $/ 10.1093 / \mathrm{gji} / \mathrm{ggx} 251$.

Bourcier, M., Bornert, M., Dimanov, A., Héripré, E., Raphanel, J.L., 2013. Multiscale experimental investigation of crystal plasticity and grain boundary sliding in synthetic halite using digital image correlation. J. Geophys. Res. Solid Earth 118, 511526. https://doi.org/10.1002/jgrb.50065.

Bystricky, M., Kunze, K., Burlini, L., Burg, J.P., 2000. High shear strain of olivine aggregates: rheological and seismic consequences. Science 290, 1564-1567. https://

doi.org/10.1126/science.290.5496.1564.

Castelnau, O., Brenner, R., Lebensohn, R.A., 2006. The effect of strain heterogeneity on the work hardening of polycrystals predicted by mean-field approaches. Acta Mater. 54, 2745-2756. https://doi.org/10.1016/j.actamat.2006.02.014.

Castelnau, O., Blackman, D.K., Lebensohn, R.A., Castañeda, P.P., 2008. Micromechanical modeling of the viscoplastic behavior of olivine. J. Geophys. Res. Solid Earth 113, 1-18.

https://doi.org/10.1029/2007JB005444.
Castelnau, O., Blackman, D.K., Becker, T.W., 2009. Numerical simulations of texture development and associated rheological anisotropy in regions of complex mantle flow. Geophys. Res. Lett. 36, 1-6. https://doi.org/10.1029/2009GL038027.

Chastel, Y.B., Dawson, P.R., Wenk, H.-R., Bennett, K., 1993. Anisotropic convection with implications for the upper mantle. J. Geophys. Res. 98, 17757-17771. https://doi. org/10.1029/93JB01161.

Chopra, P.N., Paterson, M.S., 1984. The role of water in the deformation of dunite. J. Geophys. Res. 89, 7861-7876. https://doi.org/10.1029/JB089iB09p07861.

Christensen, U., 1987. Some geodynamical effects of anisotropic viscosity. Geophys. J. R. Astr. Soc. 91, 711-736. https://doi.org/10.1111/j.1365-246X.1987.tb01666.x.

Demouchy, S., Tommasi, A., Barou, F., Mainprice, D., Cordier, P., 2012. Deformation of olivine in torsion under hydrous conditions. Phys. Earth Planet. Inter. 202-203, 56-70. https://doi.org/10.1016/j.pepi.2012.05.001.

Detrez, F., Castelnau, O., Cordier, P., Merkel, S., Raterron, P., 2015. Effective viscoplastic behavior of polycrystalline aggregates lacking four independent slip systems inferred from homogenization methods; application to olivine. J. Mech. Phys. Solids 83, 199-220. https://doi.org/10.1016/j.jmps.2015.05.022.

Durinck, J., Carrez, P., Cordier, P., 2007. Application of the Peierls-Nabarro model to dislocations in forsterite. Eur. J. Mineral. 19, 631-639. https://doi.org/10.1127/0935-1221/2007/0019-1757.

Hansen, L.N., Zimmerman, M.E., Kohlstedt, D.L., 2012a. Laboratory measurements of the viscous anisotropy of olivine aggregates. Nature 492, 415-418. https://doi.org/10. 1038/nature11671.

Hansen, L.N., Zimmerman, M.E., Kohlstedt, D.L., 2012b. The influence of microstructure on deformation of olivine in the grainboundary sliding regime. J. Geophys. Res. 117, 1-17. https://doi.org/10.1029/2012JB009305.

Hansen, L.N., Zimmerman, M.E., Dillman, A.M., Kohlstedt, D.L., 2012c. Strain localization in olivine aggregates at high temperature: a laboratory comparison of constant- strain-rate and constant-stress boundary conditions. Earth Planet. Sci. Lett. 333-334, 134-145. https://doi.org/10.1016/j.epsl.2012.04.016.

Hansen, L.N., Zhao, Y.H., Zimmerman, M.E., Kohlstedt, D.L., 2014. Protracted fabric evolution in olivine: implications for the relationship among strain, crystallographic fabric, and seismic anisotropy. Earth Planet. Sci. Lett. 387, 157-168. https://doi.org/ 10.1016/j.epsl.2013.11.009.

Hansen, L.N., Warren, J.M., Zimmerman, M.E., Kohlstedt, D.L., 2016a. Viscous anisotropy of textured olivine aggregates, Part 1: measurement of the magnitude and evolution of anisotropy. Earth Planet. Sci. Lett. 445, 92-103. https://doi.org/10.1016/j.epsl. 2016.04.008.

Hansen, L.N., Conrad, C.P., Boneh, Y., Skemer, P., Warren, J.M., Kohlstedt, D.L., 2016b. Viscous anisotropy of textured olivine aggregates: 2. Micromechanical model. J. Geophys. Res. Solid Earth 121, 7137-7160. https://doi.org/10.1002/2016JB013240. 
Honda, S., 1986. Strong anisotropic flow in a finely layered asthenosphere. Geophys. Res. Lett. 13, 1454-1457. https://doi.org/10.1029/GL013i013p01454.

Jeffery, G.B., 1922. The motion of ellipsoidal particles immersed in a viscous fluid. Proc. Royal Soc. A 102. https://doi.org/10.1098/rspa.1922.0078.

Knoll, M., Tommasi, A., Logé, R.E., Signorelli, J.W., 2009. A multiscale approach to model the anisotropic deformation of lithospheric plates. Geochem. Geophys. Geosyst. 10, 1-18. https://doi.org/10.1029/2009GC002423.

Kumazawa, M., Anderson, O.L., 1969. Elastic moduli, pressure derivatives, and temperature derivatives of single-crystal olivine and single-crystal forsterite. J. Geophys. Res. 74, 5961-5972. https://doi.org/10.1029/JB074i025p05961.

Lebensohn, R.A., Tomé, C.N., 1993. A self-consistent anisotropic approach for the simulation of plastic deformation and texture development of polycrystals: application to zirconium alloys. Acta Metall. Mater. 41, 2611-2624. https://doi.org/10.1016/09567151(93)90130-K.

Lebensohn, R.A., Ponte Castañeda, P., Brenner, R., Castelnau, O., 2011. Full- field vs. homogenization methods to predict microstructure-property relations for polycrystalline materials. In: Computational Methods for Microstructure-Property Relationships. Springer, New York, pp. 393-441.

Lebensohn, R.A., Kanjarla, A.K., Eisenlohr, P., 2012. An elastoviscoplastic formulation based on fast Fourier transforms for the prediction of micromechanical fields in polycrystalline materials. Int. J. Plast. 32-33, 59-69. https://doi.org/10.1016/j. ijplas.2011.12.005

Lev, E., Hager, B.H., 2008. Rayleigh-Taylor instabilities with anisotropic lithospheric viscosity. Geophys. J. Int. 173, 806-814. https://doi.org/10.1111/j.1365-246X. 2008.03731.x.

Lev, E., Hager, B.H., 2011. Anisotropic viscosity changes subduction zone thermal structure. Geochem. Geophys. Geosyst. 12, 1-9. https://doi.org/10.1029/2010GC003382.

Mainprice, D., Bachmann, F., Hielcher, R., Schaeben, H., 2014. Descriptive tools for the analysis of texture projects with large datasets using MTEX - Strength, symmetry and components. In: Faulkner, D.R., Mariani, E., Mecklenburgh, J. (Eds.), Rock Deformation from Field, Experiments and Theory: A Volume in Honour of Ernie Rutter, Spec. Publ. Geol. Soc., London, U. K., pp. 409 http://dx.doi.org/10.1144/ SP409.8.

Nicolas, A., Christensen, N.I., 1987. Formation of anisotropy in upper mantle peridotites: a review, in Composition, Structure and Dynamics of the Lithosphere-Asthenosphere System. Am. Geophys. Union Geo-Dyn. Monogr. Ser., vol. 16, pp. 111-123. https:// doi.org/10.1029/GD016.

Ponte Castañeda, P., 2002. Second-order homogenization estimates for nonlinear composites incorporating field fluctuations: Itheory. J. Mech. Phys. Solids 50, 737-757.

https://doi.org/10.1016/S0022-5096(01)00099-0.
Raterron, P., Chen, J., Li, L., Weidner, D., Cordier, P., 2007. Pressure-induced slip-system transition in forsterite: single-crystal rheological properties at mantle pressure and temperature. Am. Mineral. 92, 1436-1445. https://doi.org/10.2138/am.2007.2474.

Sachs, G., 1928. Zur Ableitung einer Fliessbedingung. Z. Ver. Dtsch. Ing. 72, 734-736.

Signorelli, J., Tommasi, A., 2015. Modeling the effect of subgrain rotation recrystallization on the evolution of olivine crystal preferred orientations in simple shear. Earth Planet. Sci. Lett. 430, 356-366. https://doi.org/10.1016/j.epsl.2015.08.018.

Thieme, M., Demouchy, S., Mainprice, D., Barou, F., Cordier, P., 2018. Stress evolution and associated microstructure during transient creep of olivine at $1000-1200{ }^{\circ} \mathrm{C}$. Phys. Earth Planet. Inter. 278, 34-46. https://doi.org/10.1016/j.pepi.2018.03.002.

Tielke, J.A., Hansen, L.N., Tasaka, M., Meyers, C., Zimmerman, M.E., Kohlstedt, D.L., 2016. Observations of grain size sensitive power law creep of olivine aggregates over a large range of latticepreferred orientation strength. J. Geophys. Res. 121, 506-516. https://doi.org/10.1002/2015JB012302.

Tommasi, A., Mainprice, D., Canova, G., Chastel, Y., 2000. Viscoplastic self-consistent and equilibrium-based modeling of olivine lattice preferred orientations: implications for the upper mantle seismic anisotropy. J. Geophys. Res. 105, 7893-7908. https://doi. org/10.1029/1999JB900411.

Tommasi, A., Vauchez, A., 2001. Continental rifting parallel to ancient collisional belts: an effect of the mechanical anisotropy of the lithospheric mantle. Earth Planet. Sci. Lett. 185, 199-210. https://doi.org/10.1016/S0012-821X(00)00350-2.

Tommasi, A., Knoll, M., Vauchez, A., Signorelli, J.W., Thoraval, C., Logé, R., 2009. Structural reactivation in plate tectonics controlled by olivine crystal anisotropy. Nat. Geosci. 2, 423-427. https://doi.org/10.1038/nge0528.

Tommasi, A., Vauchez, A., 2015. Heterogeneity and anisotropy in the lithospheric mantle. Tectonophysics 661, 11-37. https://doi.org/10.1016/j.tecto.2015.07.026.

Tommasi, A., Tiko , B., Vauchez, A., 1999. Upper mantle tectonics: Three-dimensional deformation, olivine crystallographic fabrics and seismic properties. Earth Planet. Sci. Lett. 168, 173186. https://doi.org/10.1016/S0012-821X(99)00046-1.

Vauchez, A., Tommasi, A., Mainprice, D., 2012. Faults (shear zones) in the Earth's mantle. Tectonophysics 558-559, 1-27. https://doi.org/10.1016/j.tecto.2012.06.006.

Vauchez, A., Tommasi, A., Barruol, G., 1998. Rheological heterogeneity, mechanical anisotropy and deformation of the continental lithosphere. Tectonophysics 296, 61-86. https://doi.org/10.1016/S0040-1951(98)00137-1.

Wenk, H.R., Bennett, K., Canova, G.R.A., Molinari, 1991. Modelling plastic deformation of peridotite with the self-consistent theory. J. Geophys. Res. 96, 8337-8349. https:// doi.org/10.1029/91JB00117.

Wenk, H.R., Armann, M., Burlini, L., Kunze, K., Bortolotti, M., 2009. Large strain shearing of halite: experimental and theoretical evidence for dynamic texture changes. Earth Planet. Sci. Lett. 280, 205-210. https://doi.org/10.1016/j.epsl.2009.01.036. 
Wheeler, J., 2010. Anisotropic rheology during grain boundary diffusion creep and its relation to grain rotation, grain boundary sliding and superplasticity. Philos. Mag. 37-41. https://doi.org/10.1080/14786431003636097.
Zhang, S., Karato, S.I., 1995. Lattice preferred orientation of olivine aggregates deformed in simple shear. Nature. https://doi.org/10.1038/375774a0. 
